\title{
O efeito da sibutramina na perda de peso de adolescentes obesos
}

\author{
The effect of sibutramine on weight loss in obese adolescents
}

Ruth Rocha Franco', Louise Cominato', Durval Damiani'

\begin{abstract}
RESUMO
Objetivo: Avaliar o efeito da sibutramina na perda de peso de adolescentes obesos. Sujeitos e métodos: Estudo duplo-cego placebo controlado tipo cross over com duração de 13 meses. Foram incluídos no estudo 73 adolescentes obesos de ambos os sexos com idades entre 10 e 18 anos. A avaliação antropométrica foi realizada a cada 40 dias em média. Os exames laboratoriais e de imagem foram realizados antes, no período de wash-out e ao final dos 13 meses. Resultados: A porcentagem de pacientes que perderam $10 \%$ do peso inicial no placebo foi de $46 \%$ e, no grupo sibutramina, foi de $75 \%$. Quando usaram o placebo, o peso em média se elevou em 1,61 kg, e o IMC reduziu em média $0,24 \mathrm{~kg} / \mathrm{m}^{2}$, enquanto com o uso da sibutramina o peso reduziu em média $4,47 \mathrm{~kg}$ e o IMC reduziu em média $2,38 \mathrm{~kg} / \mathrm{m}^{2} \mathrm{com} \mathrm{p}<0,001$. Conclusões: A sibutramina induziu significantemente mais perda de peso em adolescentes obesos em comparação ao placebo, sem efeitos colaterais significativos. A curva de evolução ponderal foi diferente de acordo com o momento em que a sibutramina foi introduzida. Esse achado indica que o melhor momento de introdução da sibutramina é quando a adesão começa a falhar. Arq Bras Endocrinol Metab. 2014;58(3):243-50

Descritores

Obesidade; sibutramina; adolescentes; índice de massa corporal; síndrome x; síndrome metabólica
\end{abstract}

1 Unidade de Endocrinologia Pediátrica, Instituto da Criança do Hospital das Clínicas da Faculdade de Medicina da Universidade de São Paulo (ICr/HCFMUSP), São Paulo, SP, Brasil

\begin{abstract}
Objective: To evaluate the effect of sibutramine on weight loss in obese adolescents. Subjetcs and methods: A double-blind controlled study lasting 13 months. The study included 73 obese adolescents of both sexes aged between 10 and 18 years. Laboratory tests and imaging studies were performed before, during wash-out, and at the end of 13 months. Results: The percentage of patients who lost $10 \%$ of their initial weight in the placebo group was $46 \%$, and in the sibutramine group was $75 \%$. When placebo was used, average weight rose by $1.61 \mathrm{~kg}$, and $\mathrm{BMI}$ decreased by $0.24 \mathrm{~kg} / \mathrm{m}^{2}$ whereas with the use of sibutramine, weight decreased by 4.47 $\mathrm{kg}$, and average BMI decreased, $2.38 \mathrm{~kg} / \mathrm{m}^{2}$, with $\mathrm{p}<0.001$. Conclusions: Sibutramine induced significantly more weight loss in obese adolescents compared with placebo, without significant side effects. The weight loss curve was different depending on the moment sibutramine was introduced. This finding indicates that the best time to start sibutramine is when adhesion begins to fail. Arq Bras Endocrinol Metab. 2014;58(3):243-50
\end{abstract}

\section{Keywords}

Obesity; sibutramine; adolescents; body mass index; syndrome x, metabolic syndrome

\section{INTRODUÇÃO}

\section{A} obesidade em crianças e adolescentes vem aumentando em proporções alarmantes em países desenvolvidos e também em países em desenvolvimento (1).

O panorama da obesidade no Brasil segue também a tendência mundial. Houve um aumento de 3,7\% no período $1974-1975$ para $21,7 \%$ no período 2008-2009 entre os meninos de 10 a 19 anos e de $7,6 \%$ para $19,4 \%$ entre as meninas dessa mesma faixa etária no mesmo período (2). Alguns estudos regionais mostram prevalências diferentes nas diversas regiões brasileiras. As taxas variam de $4,4 \%$ a $8,5 \%$ nas regiões menos desenvolvidas do Norte e Nordeste e taxas de $10,5 \%$ a $18 \%$ nas regiões do Sudeste (3). Um estudo realizado em Presidente Prudente, no estado de São Paulo, encontrou prevalência de 28,6\% entre os jovens de 10 a 17 anos (4). 
As taxas atuais de obesidade são reconhecidas como um problema de saúde pública em escala epidêmica tanto em adultos como em crianças e adolescentes (1). A elevada prevalência da obesidade infantil se torna cada vez mais alarmante e importante, uma vez que sobrepeso e obesidade nessa faixa etária são fortes indicadores de sua manutenção também na vida adulta. A obesidade, além de elevar o risco de doenças crônicas como diabetes tipo 2 , intolerância à glicose, síndrome metabólica, dislipidemia, hipertensão arterial e esteatose hepática, eleva consideravelmente o risco cardiovascular e as taxas de morbimortalidade (5).

Todas as comorbidades que estão associadas à obesidade têm seu tratamento principal baseado na perda de peso, mas o sucesso do tratamento clínico ainda é baixo.

O tratamento medicamentoso da obesidade ainda gera controvérsias e discussões sobre sua segurança, eficácia e benefícios na população adulta e mais ainda na população pediátrica.

Alguns autores como Berkowitz e cols. (6) e Godoy-Matos e cols. (7) realizaram estudos duplo-cegos placebo controlados avaliando o efeito da sibutramina em associação à mudança dietética comportamental para o tratamento da obesidade em adolescentes. Esses autores concluíram que a sibutramina é uma droga efetiva e que induz significativamente mais perda de peso do que o placebo. Ainda existe uma escassez de trabalhos duplo-cegos placebo controlados para avaliar o efeito da sibutramina nessa faixa etária. Após os trabalhos de Berkowitz e cols. (6) e Godoy-Matos e cols. (7), outros trabalhos também comprovaram uma perda de peso maior com o uso da sibutramina do que com o placebo. Poucos efeitos colaterais foram constatados, o que, na prática clínica, favoreceu o uso da sibutramina.

A sibutramina foi avaliada em um grande estudo multicêntrico, o Sibutramine Cardiovascular Outcomes (SCOUT) (8), que avaliou pacientes com mais de 55 anos e com doença cardiovascular e diabetes tipo 2 . Pôde-se concluir que houve aumento do risco relativo de eventos cardiovasculares em 16\% no grupo que usou sibutramina. Por essa razão a droga foi suspensa em 2010 na Europa e também foi suspensa sua produção nos Estados Unidos. No Brasil, essa droga ainda é comercializada, porém com maior rigor e vigilância.

O objetivo deste trabalho foi avaliar a efetividade e a segurança da sibutramina associada a um programa multidisciplinar para tratamento da obesidade e verificar a sua influência sobre as mudanças metabólicas laboratoriais.

\section{SUJEITOS E MÉTODOS}

O estudo foi duplo-cego placebo controlado tipo cross-over com duração de 13 meses. Os pacientes foram randomizados e iniciaram o estudo com o uso do placebo ou da sibutramina por 6 meses, depois passaram por um período de 30 dias (wash-out) sem receber medicação nem placebo e, nos seis meses seguintes, receberam sibutramina ou placebo inversamente ao que tinham iniciado o estudo.

Foram incluídos no estudo 73 adolescentes obesos de ambos os sexos com idades entre 10 e 18 anos, regularmente acompanhados no ambulatório de endocrinologia pediátrica no grupo de obesidade infantil do Instituto da Criança do Hospital das Clínicas da Faculdade de Medicina da Universidade de São Paulo (ICr/HCFMUSP). A inclusão dos pacientes foi feita por convite aos adolescentes na primeira consulta do ambulatório de obesidade e com autorização dos pais ou responsável legal. Para a inclusão inicial o paciente necessitava já ter realizado algum tratamento convencional (dietético/ comportamental) prévio, por pelo menos seis meses. Todos os pacientes e seus responsáveis legais foram informados por meio do Termo de Consentimento Livre e Esclarecido. O projeto foi previamente analisado e aprovado pela Comissão de Ética para Análise de Projetos de Pesquisa (CAPPesq) do HCFMUSP.

Foram utilizados os seguintes critérios para inclusão ao estudo: idade $\geq 10$ anos e $<18$ anos, diagnóstico de obesidade (classificação segundo a Organização Mundial de Saúde) (9), capacidade para entender as orientações, e consentimento do adolescente e do responsável legal. Os critérios de exclusão foram: problemas cardiovasculares e ou arritmias, história de anorexia, bulimia e ou transtornos psiquiátricos, hipertensão arterial, doenças crônicas prévias, uso de qualquer outra medicação que interferisse na mudança do peso, síndromes genéticas e ou atraso do desenvolvimento neuropsicomotor, glaucoma, uso de drogas ilícitas, tabaco e ou álcool, meninas grávidas ou que mantinham relação sexual sem contraceptivos. Os critérios de retirada de um paciente durante o estudo foram os seguintes: elevação da pressão arterial > do que o percentil 95 para a idade, qualquer distúrbio de comportamento encontrado após início do uso da medicação, qualquer sintoma que afetasse negativamente a rotina do paciente e fosse persistente após segunda semana do início do uso da medicação, como insônia, cefaleia intensa, ansiedade e mal-estar.

A confecção da cápsula placebo foi realizada pela equipe da farmácia hospitalar para que ficasse externa- 
mente idêntica à da cápsula da medicação. Essas cápsulas foram entregues aos pacientes em potes vedados com 30 cápsulas. A dosagem das cápsulas era de $10 \mathrm{mg}$ e a orientação era de que a medicação fosse ingerida diariamente às 10 horas da manhã.

Os pacientes foram acompanhados ambulatorialmente a cada 40 dias em média. Durante esses retornos, os pacientes passaram por avaliação nutricional, avaliação médica e orientação para realização de atividade física com o educador físico. A orientação dietética proposta foi de uma dieta hipocalórica com restrição de $25 \%$ do total de calorias recomendado para a faixa etária de um adolescente eutrófico. A consulta foi realizada pelo pediatra endocrinologista. Durante a consulta foram realizadas as medidas antropométricas como: peso e estatura para cálculo do índice de massa corporal (IMC), medida de pressão arterial (em repouso de 5 minutos e com manguito adequado para a circunferência do braço), circunferência abdominal (no maior diâmetro), circunferência da cintura (no menor diâmetro), circunferência do braço (no terço médio do braço esquerdo). Os efeitos adversos foram investigados sobre a forma de questionários preestabelecidos e descritos voluntariamente pelo paciente em cada retorno.

Os exames laboratoriais e de imagem foram realizados em três momentos do estudo: no início do estudo, durante o período de wash-out e ao final dos 13 meses de acompanhamento. Os exames laboratoriais foram realizados após 12 horas de jejum e constaram das dosagens séricas de: colesterol total e frações, triglicérides, leptina, proteína C reativa, transaminases (TGO e TGP) e teste de tolerância oral à glicose com 1,75 grama de glicose por kg - máx $75 \mathrm{~g}$ (com dosagens de glicose e insulina nos seguintes tempos: $0,30,60,90$, 120 minutos). Os exames de imagem realizados foram ultrassom de abdome, ecocardiograma com Doppler colorido e eletrocardiograma.

A análise estatística para as variáveis quantitativas é apresentada em tabelas com média, desvio-padrão e intervalo de confiança de $95 \%$ para as médias. Para as variáveis qualitativas frequências absolutas $(\mathrm{N})$ e relativas (\%), foi aplicado o teste de Kolmogorov-Smirnov (10) para diferenciar se as variáveis tinham distribuição normal ou não. Quando a variável apresentou distribuição normal, o teste usado foi o teste $t$-student (11) e, para as variáveis de distribuição não normal, o teste usado foi o não paramétrico de Mann-Whitney (12). Para a comparação da redução de IMC entre os grupos, foi utilizado o teste $t$-student, e a modelagem da curva de evolução ponderal foi realizada por modelos lineares mistos (12).

Os resultados dos exames laboratoriais foram testados por meio do teste $t$-student se havia diferença significativa entre as variações dos grupos e correlacionouse com a variação de IMC por meio do coeficiente de correlação de Pearson (13).

O nível de significância aplicado nos testes foi de $5 \%$, sempre considerando hipóteses alternativas bicaudais. Os softwares utilizados para realizar a análise foram o R 2.11.0 (pacote nlme para o modelo linear misto) e o Microsoft Office Excel e SPSS versão 15 (IBM).

\section{RESULTADOS}

Foram selecionados para o estudo 73 pacientes, dos quais oito desistiram da participação do estudo e não retornaram para a segunda consulta.

Foram randomizados 63 pacientes, sendo 28 do sexo masculino e 35 do sexo feminino com média de idade de 12,9 $\pm 1,78$ anos. As características dessa população são apresentadas na tabela 1 .

Dos 63 pacientes que iniciaram o estudo, somente 23 pacientes completaram todo o estudo (13 meses). Os outros 40 pacientes realizaram somente uma parte do estudo (de 5 a 10 meses), pois desistiram de manter o acompanhamento. Para melhor representação, a análise foi feita de duas formas: com todos os 63 pacientes e somente com os 23 pacientes.

\section{Variação no IMC, peso e medidas antropométricas}

Para os 23 pacientes que completaram todo o estudo, os resultados evidenciaram uma diminuição no IMC nos primeiros seis meses do estudo em torno de $2,2 \pm 3,0$ $\mathrm{kg} / \mathrm{m}^{2}$ para quem usou sibutramina e uma diminuição de $1,2 \pm 1,7 \mathrm{~kg} / \mathrm{m}^{2}$ para os pacientes que receberam o placebo. Após seis meses da mudança de placebo para sibutramina e vice-versa, os pacientes que receberam a sibutramina diminuíram o IMC em $1,7 \pm 1,3 \mathrm{~kg} / \mathrm{m}^{2} \mathrm{e}$ os que receberam o placebo elevaram o IMC em 0,9 $\pm 2,1 \mathrm{~kg} / \mathrm{m}^{2}$. As variações do peso e do IMC ao final dos 13 meses, após uso de placebo e sibutramina, não mostraram diferença significativa. $\mathrm{O}$ grupo que iniciou com o placebo reduziu o IMC em $2,7 \pm 2,6 \mathrm{~kg} / \mathrm{m}^{2} \mathrm{e} \mathrm{o}$ grupo que iniciou com sibutramina reduziu em $1,9 \pm$ $3,5 \mathrm{~kg} / \mathrm{m}^{2}(\mathrm{p}=0,5)$. 
Tabela 1. Dados antropométricos iniciais da população avaliada

\begin{tabular}{|c|c|c|c|c|c|c|c|}
\hline \multirow{2}{*}{ Medida } & \multirow{2}{*}{ Grupo } & \multirow{2}{*}{$\mathbf{N}$} & \multirow{2}{*}{ Média } & \multirow{2}{*}{ Desvio-padrão } & \multicolumn{2}{|c|}{ IC p/ médica (95\%) } & \multirow{2}{*}{ p-valor } \\
\hline & & & & & Inf. & Sup. & \\
\hline \multirow{3}{*}{ Idade (anos) } & Todos & 63 & 12,9 & 1,8 & 12,4 & 13,3 & \multirow{3}{*}{0,03} \\
\hline & $P$ & 27 & 12,3 & 1,7 & 11,7 & 13,0 & \\
\hline & S & 36 & 13,3 & 1,8 & 12,7 & 13,9 & \\
\hline \multirow{3}{*}{ Estatura (cm) } & Todos & 63 & 158,3 & 9,0 & 156,0 & 160,6 & \multirow{3}{*}{0,85} \\
\hline & $P$ & 27 & 158,5 & 10,2 & 154,5 & 162,6 & \\
\hline & $S$ & 36 & 158,1 & 8,1 & 155,3 & 160,9 & \\
\hline \multirow{3}{*}{ Peso $(\mathrm{kg})$} & Todos & 63 & 84,5 & 21,6 & 79,1 & 90,0 & \multirow{3}{*}{0,64} \\
\hline & $P$ & 27 & 83,1 & 19,6 & 75,3 & 90,9 & \\
\hline & $S$ & 36 & 85,5 & 23,2 & 77,7 & 93,4 & \\
\hline \multirow{3}{*}{ IMC $\left(\mathrm{kg} / \mathrm{m}^{2}\right)$} & Todos & 62 & 33,4 & 6,6 & 31,7 & 35,1 & \multirow{3}{*}{0,50} \\
\hline & $P$ & 27 & 32,8 & 5,8 & 30,5 & 35,1 & \\
\hline & S & 35 & 33,9 & 7,2 & 31,4 & 36,4 & \\
\hline \multirow{3}{*}{ PAS (mmHg) } & Todos & 63 & 113,1 & 8,5 & 111,0 & 115,3 & \multirow{3}{*}{0,10} \\
\hline & $P$ & 27 & 111,1 & 8,5 & 107,8 & 114,5 & \\
\hline & $S$ & 36 & 114,6 & 8,3 & 111,8 & 117,5 & \\
\hline \multirow{3}{*}{ PAD (mmHg) } & Todos & 63 & 70,4 & 8,7 & 68,2 & 72,6 & \multirow{3}{*}{0,68} \\
\hline & $P$ & 27 & 70,9 & 8,5 & 67,5 & 74,3 & \\
\hline & $S$ & 36 & 70,0 & 9,0 & 67,0 & 73,1 & \\
\hline \multirow{3}{*}{ Cintura (cm) } & Todos & 63 & 93,2 & 12,2 & 90,2 & 96,3 & \multirow{3}{*}{0,58} \\
\hline & $P$ & 27 & 92,3 & 10,3 & 88,2 & 96,4 & \\
\hline & $S$ & 36 & 93,9 & 13,7 & 89,3 & 98,6 & \\
\hline \multirow{3}{*}{ Quadril (cm) } & Todos & 63 & 109,2 & 14,0 & 105,7 & 112,7 & \multirow{3}{*}{0,55} \\
\hline & $P$ & 27 & 108,0 & 14,0 & 102,5 & 113,5 & \\
\hline & $S$ & 36 & 110,1 & 14,0 & 105,4 & 114,9 & \\
\hline \multirow{3}{*}{ Abdome (cm) } & Todos & 62 & 106,8 & 14,1 & 103,2 & 110,4 & \multirow{3}{*}{0,72} \\
\hline & $P$ & 27 & 106,1 & 12,9 & 101,04 & 111,2 & \\
\hline & $S$ & 35 & 107,3 & 15,2 & 102,1 & 112,6 & \\
\hline
\end{tabular}

Teste t-student. P: placebo; S: sibutramina; IMC: índice de massa corporal; PAS: pressão arterial sistólica; PAD: pressão arterial diastólica.

A figura 1 representa a evolução do IMC durante todo o estudo no grupo que iniciou com placebo e terminou com sibutramina (grupo A) e no grupo que iniciou com sibutramina e terminou com o placebo (grupo B). A figura 2 representa a comparação dos dois grupos (A e B) durante todo o período do estudo.

A análise dos 63 pacientes, nos seis meses iniciais de intervenção, evidenciou ganho de $10 \%$ no peso inicial em $54 \%$ dos pacientes que receberam o placebo, enquanto nenhum paciente que usou sibutramina apresentou ganho de peso.

A perda de $10 \%$ do peso inicial ocorreu em $46 \%$ dos indivíduos que receberam o placebo e em $75 \%$ dos indivíduos que receberam a sibutramina.
Uma perda de peso mais significativa entre $10 \% \mathrm{e}$ $16 \%$ do peso inicial ocorreu em um quarto dos pacientes que usaram sibutramina e não ocorreu em nenhum paciente que recebeu o placebo.

Ao término dos primeiros seis meses, o grupo que iniciou com placebo elevou o peso em média $1,6 \pm$ $4,5 \mathrm{~kg}$ e o IMC reduziu em média $0,2 \pm 1,6 \mathrm{~kg} / \mathrm{m}^{2}$, já o grupo que iniciou com sibutramina reduziu o peso em média $4,4 \pm 5,9 \mathrm{~kg}$ e o IMC em média $2,4 \pm 2,4$ $\mathrm{kg} / \mathrm{m}^{2}(\mathrm{p}<0,001)$. No período do $7^{\circ}$ mês ao $13^{\circ}$ mês do estudo, o grupo que usou a sibutramina reduziu o peso em $1,7 \pm 2,5 \mathrm{~kg}$ e o IMC em $1,4 \pm 1,3 \mathrm{~kg} / \mathrm{m}^{2}$, e o grupo que usou o placebo elevou o peso em $4,4 \pm 5,2$ $\mathrm{kg}$ e o IMC em $0,9 \pm 1,9 \mathrm{~kg} / \mathrm{m}^{2}(\mathrm{p}<0,001)$. 


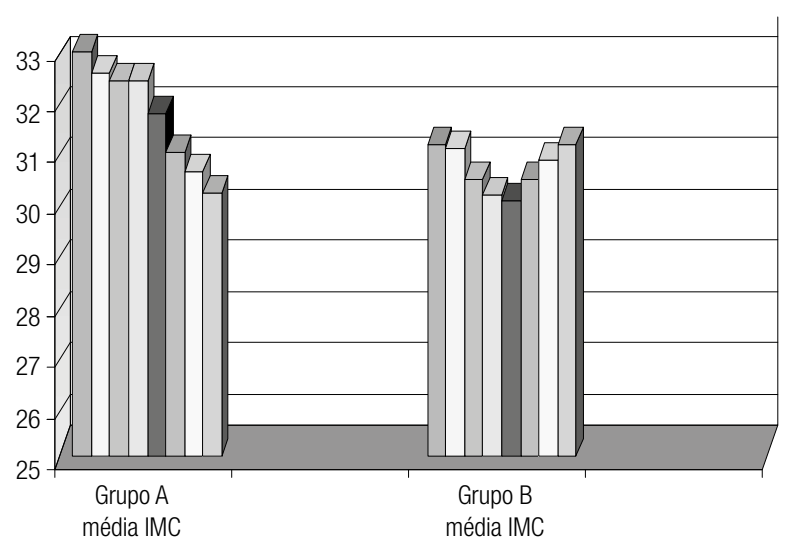

$\square$ IMC Inicial $\square$ IMC Cons $2 \square$ IMC Cons $3 \square$ IMC Cons 4

$\square$ Washout IMC $\square$ IMC Cons $6 \square$ IMC Cons $7 \square$ IMC Cons 8

IMC: índice de massa corporal; CONS: consulta; Grupo A: início com placebo, após washout recebeu sibutramina; Grupo B: início com sibutramina, após washout recebeu placebo.

Figura 1. Evolução do IMC durante todo o estudo nos 23 pacientes aderentes

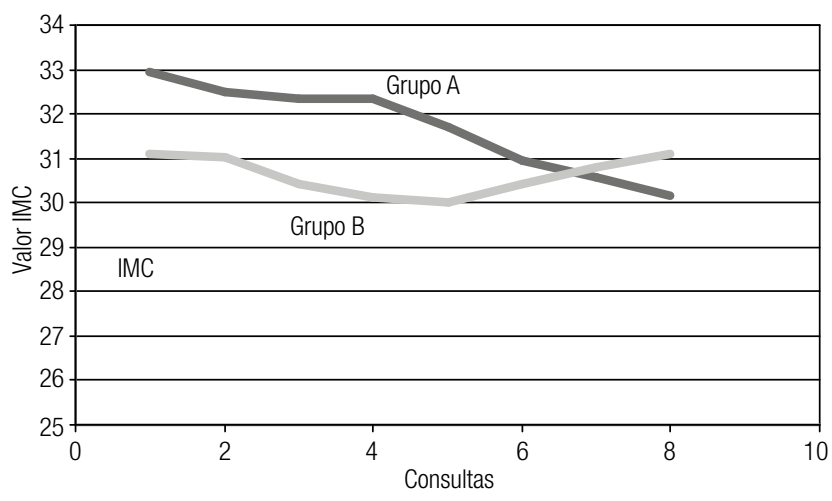

IMC: índice de massa corporal; CONS: consulta; Grupo A: início com placebo, após washout recebeu sibutramina; Grupo B: início com sibutramina, após washout recebeu placebo.

Figura 2. Comparação da evolução do IMC nos grupos A e B

Em relação às medidas antropométricas, todas diminuíram significativamente acompanhando a perda de peso. A circunferência abdominal diminuiu em média $3,7 \pm 5,0 \mathrm{~cm}$ no grupo sibutramina e elevou $0,6 \pm 4,9 \mathrm{~cm}$ no grupo placebo no primeiro período do estudo $(\mathrm{p}=$ $0,01)$.

As características como idade, sexo, raça e maturidade sexual não tiveram impacto nas mudanças de IMC em ambos os grupos. Não houve diferença significativa nos níveis de pressão arterial entre os grupos placebo e sibutramina.

\section{Mudanças nos exames laboratoriais e de imagem}

Para os 23 pacientes que realizaram as dosagens laboratoriais nos momentos adequados do estudo, a análise dos dados evidenciou uma tendência à diminuição no nível de colesterol total, LDL-colesterol, triglicérides, insulina, leptina e PCR no momento em que usaram a sibutramina, assim como uma tendência à elevação do HDL colesterol, porém sem diferença estatística. Os ecocardiogramas realizados durante o estudo não evidenciaram nenhuma mudança e ou anormalidade morfológica valvar e disfunção diastólica nos grupos estudados. Não houve mudança no padrão ultrassonográfico da região hepática durante as três avaliações feitas durante o estudo (Tabela 2).

Tabela 2. Evolução dos parâmetros laboratoriais inicial e final do primeiro período

\begin{tabular}{|c|c|c|c|}
\hline Exames & $\begin{array}{l}\text { Sibutramina } \\
\text { Média/DP }\end{array}$ & $\begin{array}{c}\text { Placebo } \\
\text { Média/DP }\end{array}$ & P-valor \\
\hline Colesterol inicial & $158,1 \pm 28,1$ & $158,6 \pm 29,6$ & 0,96 \\
\hline Colesterol final & $154,0 \pm 35,3$ & $160,7 \pm 25,3$ & 0,52 \\
\hline Diferença & $-4,1 \pm 26,2$ & $2,0 \pm 29,2$ & 0,50 \\
\hline LDL inicial & $102,9 \pm 27,54$ & $99,0 \pm 26,6$ & 0,66 \\
\hline LDL final & $93,2 \pm 25,8$ & $98,6 \pm 20,4$ & 0,49 \\
\hline Diferença & $-9,8 \pm 17,4$ & $0,4 \pm 23,8$ & 0,14 \\
\hline HDL inicial & $39,0 \pm 7,8$ & $42,4 \pm 8,8$ & 0,21 \\
\hline HDL final & $43,7 \pm 10,0$ & $43,1 \pm 9,5$ & 0,85 \\
\hline Diferença & $4,7 \pm 10,7$ & $0,1 \pm 10,2$ & 0,19 \\
\hline TG inicial & $83,1 \pm 38,3$ & $87,5 \pm 37,8$ & 0,72 \\
\hline TG final & $67,5 \pm 35,6$ & $92,1 \pm 45,3$ & 0,07 \\
\hline Diferença & $-15,6 \pm 26,0$ & $4,5 \pm 50,0$ & 0,12 \\
\hline PCR inicial & $7,4 \pm 17,5$ & $3,6 \pm 2,6$ & 0,39 \\
\hline PCR final & $2,3 \pm 4,1$ & $3,8 \pm 3,0$ & 0,23 \\
\hline Diferença & $-5,1 \pm 17,2$ & $0,3 \pm 2,6$ & 0,24 \\
\hline Leptina inicial & $14,6 \pm 12,7$ & $18,9 \pm 14,6$ & 0,34 \\
\hline Leptina final* & $12,1 \pm 7,9$ & $22,9 \pm 22,9$ & 0,05 \\
\hline Diferença & $-1,7 \pm 12,9$ & $4,4 \pm 23,8$ & 0,33 \\
\hline TGO inicial & $26,6 \pm 15,8$ & $25,6 \pm 12,1$ & 0,82 \\
\hline TGO final & $23,2 \pm 9,9$ & $22,2 \pm 8,4$ & 0,74 \\
\hline Diferença & $-3,4 \pm 13,7$ & $-3,9 \pm 9,3$ & 0,89 \\
\hline TGP inicial & $35,4 \pm 8,0$ & $40,5 \pm 16,9$ & 0,23 \\
\hline TGP final & $33,5 \pm 11,4$ & $38,0 \pm 9,3$ & 0,20 \\
\hline Diferença & $-1,9 \pm 9,1$ & $3,1 \pm 13,4$ & 0,75 \\
\hline Glicemia inicial & $82,5 \pm 20,3$ & $76,4 \pm 30,3$ & 0,47 \\
\hline Glicemia final & $78,57 \pm 26,6$ & $81,1 \pm 21,6$ & 0,74 \\
\hline Diferença & $-3,9 \pm 32,0$ & $4,7 \pm 37,1$ & 0,45 \\
\hline Insulina inicial & $20,9 \pm 22,5$ & $16,9 \pm 9,6$ & 0,50 \\
\hline Insulina final & $12,1 \pm 15,9$ & $16,3 \pm 8,8$ & 0,33 \\
\hline Diferença & $-8,8 \pm 21,0$ & $0,6 \pm 12,1$ & 0,17 \\
\hline
\end{tabular}

TGO: transaminase oxalacética; TGP: transaminase pirúvica; TG: triglicérides; PCR: proteína C reativa. ${ }^{*} p<0,05$. 


\section{Efeitos adversos}

Os eventos adversos foram relatados em $18,3 \%$ do total de todas as consultas, sendo $4,9 \%$ no grupo placebo e $13,4 \%$ no grupo sibutramina $(\mathrm{p}<0,001)$.

Os efeitos adversos de maior frequência foram cefaleia e diarreia no grupo placebo e cefaleia e constipação no grupo sibutramina. Demais sintomas descritos e suas frequências estão descritos na tabela 3 .

Tabela 3. Frequência dos sintomas

\begin{tabular}{lccc}
\hline Sintomas & $\begin{array}{c}\text { Placebo } \\
(\mathbf{\%})\end{array}$ & $\begin{array}{c}\text { Sibutramina } \\
\mathbf{( \% )}\end{array}$ & $\begin{array}{c}\text { Total consultas } \\
\text { (\%) }\end{array}$ \\
\hline Alteração do paladar & 0,9 & 0 & 0,4 \\
Anorexia & 0 & 0,9 & 0,4 \\
Boca seca* & 0 & 1,7 & 0,9 \\
Cefaleia & 3,3 & 6,8 & 5,1 \\
Constipação* & 0 & 3,8 & 2,0 \\
Alteração do humor* & 0 & 1,3 & 0,7 \\
Diarreia** & 2,8 & 0 & 1,3 \\
Dispneia & 0 & 0,4 & 0,2 \\
Epigastralgia & 0 & 0,9 & 0,4 \\
Hipertensão & 0,5 & 0,9 & 0,7 \\
Insônia* & 0 & 1,3 & 0,7 \\
Irritabilidade* & 1,4 & 0 & 0,7 \\
Náusea* & 0 & 2,1 & 1,1 \\
Taquicardia & 0,5 & 1,3 & 0,9 \\
Tontura & 0,9 & 3,4 & 2,2 \\
Tremores & 0 & 0,4 & 0,2 \\
Vômitos & 0 & 0,4 & 0,2 \\
\hline * & & &
\end{tabular}

${ }^{*} p<0,05 ;{ }^{* *} p<0,01$.

\section{DISCUSSÃO}

Este foi o primeiro estudo duplo-cego placebo controlado com delineamento tipo cross-over realizado no Brasil. Esse tipo de estudo tem a vantagem de eliminar a grande variação existente entre indivíduos em resposta a um tratamento. Neste caso, os tratamentos (placebo e medicação) foram testados nos indivíduos do estudo e cada paciente foi seu próprio controle.

Durante o desenvolvimento da pesquisa muitos fatores prejudicaram o estudo da forma como havia sido proposta inicialmente. Houve grande taxa de abandono por parte dos pacientes, falhas nos momentos corretos das coletas de exames e dos retornos programados para as avaliações antropométricas.

Muitos pacientes abandonaram o tratamento, sendo alguns por estarem desmotivados pela lenta perda de peso e outros por situações não esclarecidas. A taxa de abandono foi de $63,4 \%$, assim como em outros estudos anteriores. No estudo Sibutramine Trial of Obesity Reduction and Maintenance (STORM) (14), a taxa de abandono foi de $85 \%$ para os pacientes do grupo placebo e $50 \%$ para os pacientes do grupo sibutramina.

Os resultados de perda de peso deste estudo são similares aos resultados da literatura, mas o que pode se notar é que existiu um momento em que a sibutramina interferiu de maneira mais positiva na perda de peso. Esse fato só pode ser evidenciado quando os 23 pacientes que concluíram todo o estudo tiveram seus resultados analisados separadamente.

Quando os 63 pacientes foram avaliados até o $6^{\circ}$ mês de tratamento, pode se constatar que a porcentagem de pacientes que perderam mais do que $10 \%$ do peso inicial foi $66 \%$ maior com o uso da sibutramina do que com o uso do placebo. Esses dados podem inferir que a sibutramina melhora as condições metabólicas com a perda de peso, uma vez que uma perda a partir de $7,6 \%$ ou uma redução $>0,25 \mathrm{~kg} / \mathrm{m}^{2}$ no IMC de adolescentes já é capaz de melhorar os parâmetros metabólicos, como evidenciado nos estudos de Masquio e cols. (15) e de Ford e cols. (16).

Apesar de a sibutramina ter reduzido o peso, o IMC e a medida de circunferência abdominal, as mudanças metabólicas laboratoriais não foram constatadas neste estudo, embora a tendência à melhora tenha sido evidenciada.

Nos quatro principais estudos placebo controlados que avaliaram adolescentes obesos, a perda de peso foi significativamente maior no grupo que usou a sibutramina do que no grupo que usou o placebo. Os dados do presente estudo estão um pouco abaixo dos valores de outro estudo brasileiro conduzido por Godoy-Matos e cols. (7), que avaliou uma população etnicamente similar a este. Godoy-Matos e cols. (7) demonstraram variação de IMC no grupo que usou sibutramina de $-3,6 \pm 2,5 \mathrm{~kg} / \mathrm{m}^{2}$ e no grupo placebo de $0,9 \pm 0,9 \mathrm{~kg} / \mathrm{m}^{2}$. Os resultados deste estudo em relação à variação do IMC foram $-2,4$ $\pm 2,4 \mathrm{~kg} / \mathrm{m}^{2}$ para sibutramina e $-0,2 \pm 1,6 \mathrm{~kg} / \mathrm{m}^{2}$ para o placebo. Os valores encontrados por Berkowitz (6) estão mais próximos dos valores encontrados neste estudo.

A perda de peso neste estudo foi inferior à do estudo de Godoy-Matos e cols. (7) e de outros autores como Berkowitz e cols. (6-17), Violante-Ortìz e cols. (18) e García-Morales e cols. (19).

Para aqueles 23 pacientes que completaram todo o estudo corretamente, sem falhas, os resultados evi- 
denciaram que eles perderam peso nos primeiros seis meses independentemente de usar sibutramina ou placebo. Nos seis meses subsequentes, a sibutramina foi decisiva na manutenção da perda de peso. Os pacientes que receberam primeiro a sibutramina e passaram a receber o placebo posteriormente não conseguiram manter a perda de peso. Os pacientes que receberam o placebo primeiro e passaram a receber a sibutramina no segundo momento do estudo continuaram a perder peso. Supõe-se que esse fato tenha ocorrido porque esse grupo de pacientes que completaram todo o estudo foi o de melhor aderência e seguiu as orientações dietéticas e comportamentais corretamente e por essa razão perdeu peso, mesmo quando usou o placebo nos primeiros seis meses do estudo. No segundo momento do estudo (iniciado no $7^{\circ}$ mês de tratamento), esses pacientes não sustentaram o mesmo empenho e dedicação para manter as mudanças dietético-comportamentais e, dessa forma, ao receberem o placebo não mantiveram a perda de peso, já os que receberam a sibutramina reduziram o peso.

A sibutramina teve uma importância na perda de peso em ambos os períodos, porém foi mais evidente nos seis meses finais, quando a aderência às mudanças dietético-comportamentais começou a falhar.

Esse fármaco pode ser considerado um bom coadjuvante na perda de peso, principalmente nos momentos em que a aderência não estiver satisfatória.

Não houve diferença estatística na perda de peso e redução do IMC em começar o estudo fazendo uso da sibutramina ou do placebo. O efeito da redução do IMC é invertido após o wash-out e os pacientes reduziram o IMC ao final do estudo em torno de $2,2 \mathrm{~kg} / \mathrm{m}^{2}$ para aqueles que começaram com sibutramina e terminaram com o placebo ou vice-versa.

A sibutramina não mantém seu efeito após a parada de sua administração, de modo que, se o paciente não modificar seus hábitos, os benefícios conseguidos se perderão no tempo.

Quanto aos efeitos colaterais mais temidos, aumento da frequência cardíaca e da pressão arterial, esses são geralmente compensados pela perda de peso e redução do IMC e isso parece ser melhor em adolescentes do que em adultos (20). Neste estudo, a frequência de efeitos colaterais durante o uso da sibutramina foi 2,7 vezes maior em relação ao placebo. Os efeitos colaterais com significância estatística foram: constipação, boca seca, alteração de humor e insônia no grupo sibutramina e diarreia e irritabilidade no grupo placebo.
Não houve efeitos colaterais graves nem elevação dos níveis de pressão arterial.

A obesidade na adolescência é um sério problema de saúde pública com consequências físicas e psicossociais significantes, além de ser um fator de risco para morbimortalidade na vida adulta. $\mathrm{O}$ tratamento é essencial para ajudar a reduzir tais complicações. $\mathrm{O}$ tratamento convencional sem medicação em adolescentes leva a uma modesta perda de peso como alguns trabalhos têm mostrado (21).

$\mathrm{O}$ uso de agentes farmacológicos como coadjuvante no tratamento da obesidade na adolescência ainda é alvo de críticas, apesar de ter seus resultados positivos e benefícios claramente comprovados na literatura.

Algumas lacunas acerca desse tema ainda faltam ser esclarecidas e devem ser mais bem avaliadas. A questão do melhor momento para se iniciar uma medicação antiobesidade durante o tratamento é uma delas. Neste estudo, pode se esclarecer que o melhor momento é durante a fase em que ocorrem desmotivação e não sustentação às mudanças comportamentais. No início do tratamento, os pacientes estavam mais motivados e, mesmo fazendo uso do placebo, também conseguiram perder peso, porém nos seis meses finais esse fato não ocorreu.

A sibutramina mostrou ser um forte aliado no tratamento da obesidade, principalmente no momento em que as mudanças dietético-comportamentais não são mantidas a longo prazo.

Agradecimentos: aos pacientes e toda equipe envolvida - médicos, enfermeiros, farmacêuticos, nutricionistas, psicólogos e educadores físicos -, pela concretização desta pesquisa.

Declaração: os autores declaram não haver conflitos de interesse científico neste estudo.

\section{REFERÊNCIAS}

1. Gupta N, Goel K, Shah P, Misra A. Childhood obesity in developing countries: epidemiology, determinants, and prevention. Endocr Rev. 2012;33(1):48-70.

2. Instituto Brasileiro de Geografia e Estatística (IBGE). Pesquisa de Orçamentos Familiares (POF) 2008-2009 [citada 2010 Set 5]. Disponível em: http://www.ibge.gov.br/home/presidencia/noticias/noticia_visualiza.php?id_noticia=1699\&id_pagina=1. Acesso em: 21 fev, 2010.

3. Duncan S, Duncan EK, Fernandes RA, Buonani C, Bastos KDN, Segatto AFM, et al. Modifiable risk factors for overweight and obesity in children and adolescents from São Paulo, Brazil. BMC Public Health. 2011;11:585.

4. Fernandes RA, Kawaguti SS, Agostini L, de Oliveira AR, Ronque ERV, Freitas If Jr. Prevalence of overweight and obesity among students at private schools in Presidente Prudente, SP, Brazil. Rev Bras Cineantropom Desempenho Hum. 2007;9(1):21-7. 
5. Speiser PW, Rudolf MC, Anhalt H, Camacho-Hubner C, Chiarelli F, Eliakim A, et al. Childhood obesity. J Clin Endocrinol Metab. 2005;90(3):1871-87.

6. Berkowitz RI, Fujioka K, Daniels SR, Hoppin AG, Owen S, Perry AC, et al.; Sibutramina Adolescent Stugy Group. Effects of sibutramine treatment in obese adolescents: a randomized trial. Ann Inten Med. 2006;145(2):81-90.

7. Godoy-Matos A, Carraro L, Vieira A, Oliveira J, Guedes EP, Mattos $L$, et al. Treatment of obese adolescents with sibutramine: a randomized, double-blind, controlled Study. J Clin Endodrinol Metab. 2005;90:1460-5.

8. James WP, Caterson ID, Coutinho W, Finer N, Van Gaal LF, Maggioni AP, et al. Effect of sibutramine on cardiovascular outcomes in overweight and obese subjects. N Engl J Med. 2010;363(10):905-17.

9. World Health Organization. The use and interpretation of antropometry. Geneva:WHO; 1995.

10. Siegel S, Castellan NJ. Nonparametric statistics. 2.ed. New York: McGraw-Hill; 1998.

11. Neter J, Kutner M, Nachtsheim CJ, Wasserman W. Applied Linear Statistical Models. 4.ed. U.S.A.Times Mirror Higher Education Group; 1996.

12. Pinheiro JC, Bates DM. Mixed-effects models in S and S-PLUS. Springer, 2000.

13. Venables WN, Ripley BD. Modern Applied Statistics with S. 4.ed. Springer-Verla; 2002.

14. James WP, Astrup A, Finer N, Hilsted J, Kopelman P, Rössner $S$, et al. Effect of sibutramine on weight maintenance after weight loss: a randomised trial. STORM Study Group. Sibu- tramine Trial of Obesity Reduction and Maintenance. Lancet. 2000;356(248):2119-25.

15. Masquio DCL, Piano A, Sanches LP, Corgosinho FC, Campos RMS, Carnier J, et al. The effect of weight loss magnitude on pro-anti-inflammatory adipokines and carotid intima-media thickness in obese adolescents engaged in interdisciplinary weight loss therapy. Clin Endocrinol. 2013;79(1):55-64.

16. Ford AL, Hunt LP, Cooper A, Shield JP. What reduction in BMI SDS is required in obese adolescents to improve body composition and cardiometabolic health? Arch Dis Child. 2010;95(4):256-61.

17. Berkowitz RI, Wadden TA, Tershakovec AM, Cronquist JL. Behavior therapy and sibutramine for the treatment of adolescent obesity: a randomized controlled trial. JAMA. 2003;289(14):1805-12.

18. Violante-Ortìz $R$, Del-Rio-Navarro $B E$, Lara-Esqueda $A$, Pèrez $P$, Fanghänel G, Madero $A$, et al. Use of sibutramine in obese Hispanic adolescents. AdvTher. 2005;22(6):642-9.

19. García-Morales LM, Berber A, Macias-Lara CC, Lucio-Ortiz C, Del-Rio-Navarro BE, Dorantes-Alvárez LM. Use of sibutramine in obese Mexican adolescents: a 6-month, randomized, double-blind, placebo-controlled, parallel-group trial. Clin Ther. 2006;28(5):770-82.

20. Daniels SR, Long B, Crow S, Styne D, Sothern M, Vargas-Rodriguez I, et al.; Sibutramine Adolescent Study Group. Cardiovascular effects of sibutramine in the treatment of obese adolescents: results of a randomized, double-blind, placebo-controlled study. Pediatrics. 2007; 120:147-57.

21. Robinson TN. Behavioural treatment of childhood and adolescents obesity. Int J Obes Relat Metab Disord. 1993;23(2S):52-7. 